\title{
MORBIDADE DA ESOUISTOSSOMOSE MANSONI EM UMA COMUNIDADE RURAL DE MINAS GERAIS *
}

\author{
Maria José Conceição e J.R. Coura
}

Realizou-se um estudo seccional sobre a esquistossomose mansoni em uma comunidade rural do Municipio de Itanhomi, Vale do Rio Doce, Minas Gerais, no periodo de 1973 a 1974, com o objetivo de determinar a morbidade da doença em 1480 habitantes da população, através da avaliação das formas clínicas, da prevalência, dos indices de infecção dos moluscos e do contato da população com as águas dos córregos.

Em 1973, a prevalência da infecção esquistossomótica através de exames de fezes foi de $60,8 \%$ e, em 1974, de 62,3\%, com predominio em jovens entre 16 e 30 anos e nos de sexo masculino.

A classificação clínica dos pacientes infectados revelou $61,7 \%$ com esquistossomose infec ção; 32,5\% com esquistossomose-doença, forma hépato-intestinal e 5,8\% com forma hépato-esp/ênica, dos quais $0,8 \%$ apresentavam hipoevolutismo.

Estabeleceu-se uma correlação direta entre as formas hépato-esplênicas, preponderantes na faixa etária entre 11 e 15 anos e no sexo masculino, com a maior exposição aos focos de B. glabrata $e$, tamberm, com a maior intensidade de infecção, determinada pelo número mediano de ovos de $S$. mansoni por grama de fezes.

\section{INTRODUÇÃO}

A esquistossomose mansoni representa um dos mais importantes problemas de saúde pública do Brasil por sua progressiva disseminação, abrangendo extensas regiões do nordeste, leste, sudeste e sul do país, onde acomete alguns milhões de brasileiros em plena fase de desenvolvimento e produtividade.

A importância médico-sanitária da doença foi bem ressaltada na estimativa recente de Freitas $^{9}$, quando o autor assinala que "embora 80 a $90 \%$ dos infectados não apresentem esquistossomose-doença, seguramente em quatro milhões de portadores do verme, pelo menos $10 \%$, ou seja, 40.000 casos são doentes, dos quais cerca de $20 \%, 80.000$ casos, evoluem para as formas graves e mortais".

Esses dados e outros obtidos previamente por Meira 24; Rodrigues da Silva 38; e Pellon e Teixeira 30,31 , justificaram o interesse de pes- quisadores no esclarecimento da distribuição geográfica e da evolução da esquistossomose, através de inquéritos parasitológicos e clínico-epidemiológicos.

Desde as comunicações pioneiras sobre a esquistossomose por Pirajá da Silva ${ }^{35,36}$, em 1908 e 1909, na Bahia, poderiam ser demarcadas duas fases do estudo dessa doença no Brasil: uma fase em que os autores se preocuparam em constatar a existência da infecção e suas manifestações clínicas; a outra, em que se propuseram a analisar o curso da infecção, visando o conhecimento dos fatores envolvidos nas variações de gravidade da doença.

Incluem-se na primeira etapa trabalhos como os de Penna, citado por Lutz19, em 1916: Lutz e Penna21; Maciel22; Martins e Versiani23; Meira24.

Rodrigues da Silva38 assinalou os focos descritos na literatura nacional e os respectivos índices de infecção.

Trabalho do Departamento de Medicina Preventiva da Faculdade de Medicina da U.F.R.J., realizado com o auxilio do Conselho de Desenvolvimento Científico e Tecnológico.

Recebido para publicação em 15.1.1978. 
Em 1950, Pellon e Teixeira determinaram a prevalência da infecção em 11 Estados da Federação, através de exames de fezes em escolares entre 7 e 14 anos, completando o inquérito nacional em 1953.

A história natural da doença começou a ser evidenciada e enriquecida por Pessoa e Barros 33 ao determinarem a prevalência e classificação clínica de 1500 habitantes em Sergipe.

Em seqüência a essa etapa, Brener e Mourão ${ }^{3}$ encetaram os primeiros trabalhos longitudinais em Minas Gerais, que foram reavaliados após 10 anos, por Katz e Brener ${ }^{15}$.

Kloetzel17 observou 119 pacientes com forma hépato-esplênica em Gameleira, Pernambuco, estudando as relações entre as formas clínicas graves e a carga parasitária (1962).

Barbosa ${ }^{1}$ analisou dados de sua observa. ção sobre a morbidade de esquistossomose em 4 localidades de Pernambuco., pesquisa esta iniciada em 1958.

Ainda em Minas Gerais, Katz e Cols ${ }^{14}$, têm desenvolvido pesquisas, na localidade, de Baldim, iniciando a aplicação de moluscicida e o tratamento dos portadores com Hycanthone.

$\mathrm{Na}$ Bahia, estão sendo estudadas longitudinalmente as áreas de Caatinga do Moura (Prata e Bina 37, e Várzea Nova (Bina e Prata 2).

Coura e Cols7, no Rio de Janeiro, a partir de 1960, vêm observando em análise progressiva 2.484 pacientes oriundos de 12 Estados do Brasil, entre os quais $13 \%$ apresentavam hépato-esplenomegalia. Notaram diferença regional de procedência dos casos com formas graves, porém fazem reserva quanto ao tipo de amostra hospitalar que consideraram viciada.

Visando um estudo de morbidade em ambiente de transmissão ativa, foram estabelecidas 3 áreas para estudos seccionais e evolutivos, uma das quais está incluída no presente trabalho. As áreas em estudo pelo nosso grupo compreendem os município de Riachuelo, em Sergipe; Itanhomi e Padre Paraíso, em Minas Gerais, consideradas, a princípio, como de diferentes morbidades.

Inquérito efetuado por Menezes e Cou$\mathrm{ra}^{25}$, na sede do município de Riachuelo, em em Sergipe, mostrou $50,5 \%$ de indivíduos infectados, dos quais apenas $2,43 \%$ com hépatoesplenomegalia.

Resultados preliminares obtidos por Santos e Coura39, no município de Padre Parai- so, em Minas Gerais, revelaram $63,7 \%$ de prevalência da infecção e 3,5\% de formas hépatoesplênicas.

Ainda com o próposito de contribuir para o conhecimento da morbidade da doença,

Conceição e cols6, iniciaram em 1972 estudo piloto em Santa Luzia do Carneiro, zona rural do Município de Itanhomi, Vale do Rio Doce, verificando-se prevalência de $42,1 \%$ e hápato-esplenomegalia em 3,2\% dos pacientes infectados.

Na área urbana do Município da Intanhomi, em Minas Gerais, Lewis e cols 18 verificaram $40,3 \%$ de infectados e 3,2\% de formas hépato-esplênicas.

Em 1973 iniciou-se, sob os auspícios do CNPq, a presente pesquisa em Capitão Andrade, comunidade rural do Município de Itanhomi, uma vez que a população de 1.480 habitantes dessa área propiciava estudos prospectivos, como os recomendados pelo plano integrado de pesquisa daquele Conselho.

$O$ presente trabalho teve os seguintes objetivos:

1. O estudo da prevalência da infecção esquistossomótica através de exames de fezes e ou intradermo-reação.

2. Estabelecer a classificação clínica da doença de acordo com sua morbidade na área.

3. Verificar a espécie vetora na área e as variações de seus índices de infecção em períodos regulares.

Planejou-se um estudo longitudinal na área, visando a reavaliação periódica das formas clínicas, do índice de transmissão e conseqüentemente da incidência da infecção e de outros fatores relacionados com a evolução da doença na área em estudo.

\section{DESCRIÇÃO DA ÁREA E DE SUA POPU- LAÇÃO}

Itanhomi constitui um dos 65 municípios que compõem o Vale do Rio Doce, em Minas Gerais. Tem por coordenadas geográficas 19010'30" latitude sul e 41052'18' longitude oeste.

Limita-se com os municípios de Caratinga, Conselheiro Pena, Governador Valadares, Tarumirim e Tumiritinga (Fig. 1).

Com área de $794 \mathrm{Km}^{2}$, apresenta segundo censo do IBGE (1970), população de $\mathbf{2 5 . 6 1 5}$ 


\section{FIG. I}



MAPA MUNCIPAL CENSITÁRIO- 1970
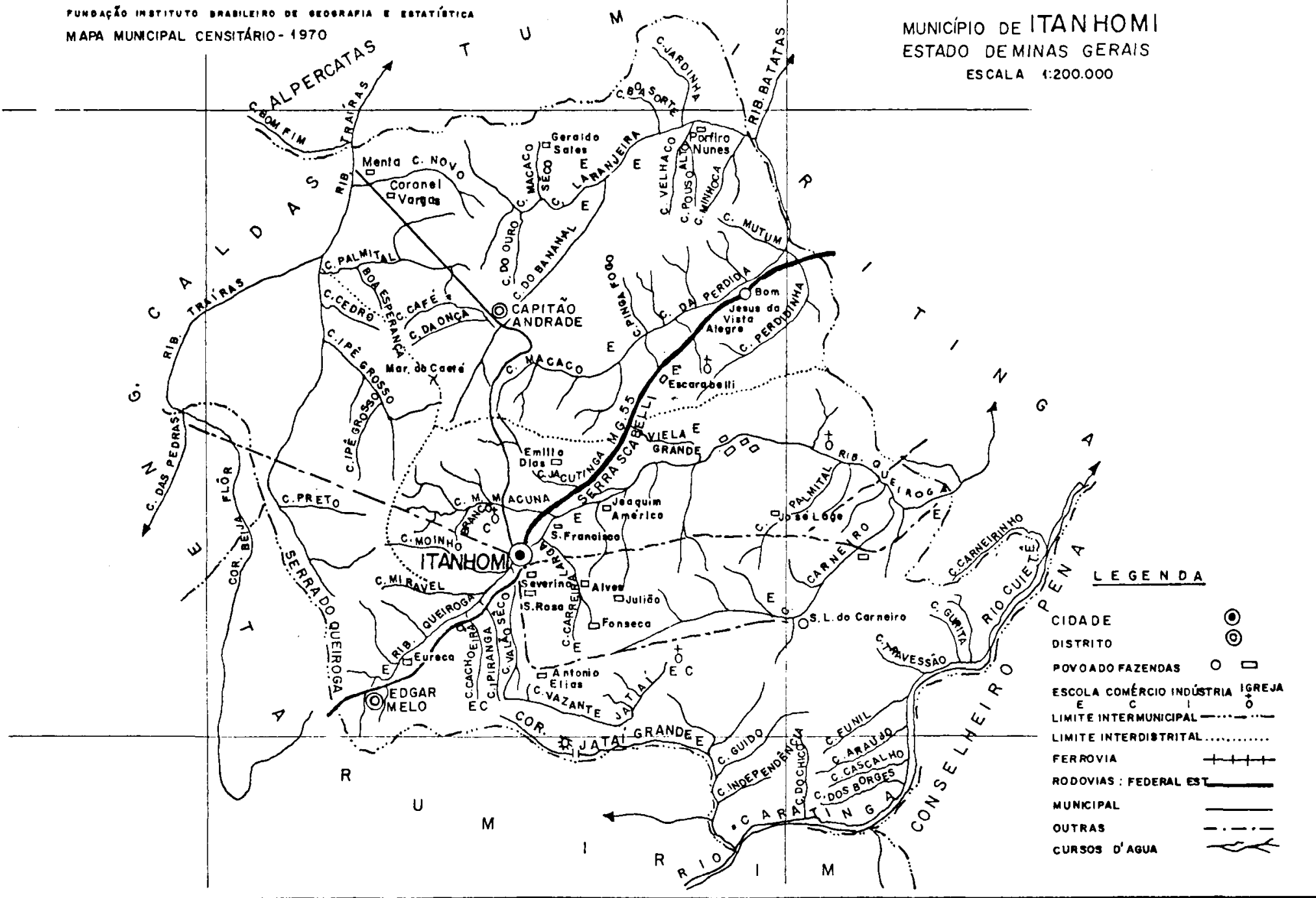
habitantes, sendo 3.334 na zona urbana e 22.281 na zona rural. A densidade demográfica é de 32,26 habitantes por $\mathrm{Km}^{2}$.

O município de Itanhomi é caracterizado por uma topografia acidentada, entrecortada por vales; está situado a $\mathbf{2 5 5}$ metros acima do nivel do mar. A precipitação pluvial média anual é de $815 \mathrm{~mm}$; apresenta clima tropical, com temperatura máxima de $40^{\circ} \mathrm{C}$, mínima de $28^{\circ} \mathrm{C}$ e média de $30^{\circ} \mathrm{C}$.

A economia do Município baseia-se na atividade agro-pecuária. As culturas agrícolas predominantes são as de milho, arroz e feijão.

Como vias de acesso ao município há duas rodovias municipais não pavimentadas que conduzem à BR-116 (Rio-Bahia), comunicando-se com Caratinga e Governador Valadares, respectivamente.

O Município está dividido em 3 distritos: Edgar de Melo, Itanhomi (sede) e Capitão Andrade, onde se procedeu este trabalho. Esse último distrito situa-se a $18 \mathrm{Km}$ da sede municipal, interligando-os estrada não asfal tada.

O distrito de Capitão Andrade é percorrido em toda sua extensão pelos córregos do Café e do Bananal (Fig. II), que se unem e vão desaguar no Rio Caratinga e este, no Rio Doce. A sede desse distrito possui 322 residências numa proporção de 4,6 pessoas por habitação. A área da sede do distrito constituía-se em 1973 de 1.480 habitantes: 730 do sexo masculino $(49,3 \%)$ e 750 do sexo feminino $(50,6 \%)$ (Gráfico I).

Quanto aos grupos étnicos, $72,7 \%$ da população era constituída de brancos, $29,3 \%$ de mestiços e $1,8 \%$ de negros.

Em virtude da emigração de 243 pessoas, ou seja, $16,4 \%$ da população, além de 19 óbitos $(1,29 \%)$, prosseguiu-se no estudo evolutivo de 1.218 dos 1.480 habitantes iniciais. A população em 1974 foi acrescida de $15 \%$ de imigrantes ( 212 pessoas) e 21 nascimentos $(1,41 \%$ ) passando a constituir-se de 1.451 habitantes.

0 distrito de Capitão Andrade possui um grupo escolar e um Jardim de Infância que matricularam, em 1974, 678 e 115 alunos, respectivamente. É desprovido de serviço médico-hospitalar, de abastecimento d'água, de rede de esgotos e de luz elétrica. A população recorre ao Hospital Municipal de Itanhomi e em casos graves os cioentes são transferidos pa- ra o Serviço Hospitalar de Governador Valadares.

\section{MATERIAL E MÉTODOS}

a) Mapeamento da área e cadastro da população.

Inicialmente mapeou-se a área a ser pesquisada, cadastrando-se as residências, após numerá-las e localizando-se no mapa as coleções de água do perímetro urbano do distrito (Fig. II). Os córregos foram subdivididos em estações, ou seja, trechos delimitados a cada cem metros nos cursos d'água, por marcos numerados, a fim de serem estabelecidos os locais de captura dos moluscos.

Em seguida realizou-se o censo da população para o acompanhamento posterior e para o estudo em sua totalidade.

\section{b) Exames coproscópicos}

Para realização dos exames coproscópicos distribuíam-se os recipientes plásticos para coleta das fezes, rotulados e identificados por meio de nomes e números, em ordem decrescente do chefe de família ao filho mais jovem e, em caso de analfabetos, o recipiente era identificado através de figuras.

Empregaram-se dois métodos diagnósticos: o método de sedimentação qualitativo de Lutz 20 , descrito posteriormente por Hoffman, Pons, Janer ${ }^{11}$; o outro método usado foi o quantitativo de Kato13, modificado por Katz, Chaves e Pellegrino ${ }^{16}$.

Das 1.234 amostras de fezes recebidas ( $83,3 \%$ da população), foram preparadas duas lâminas para cada método.

Pelo método quantitativo empregado, o peso médio estimado da amostra que preenchia o furo do cartão foi de $43,7 \mathrm{mg}$, daí ter-se calculado o número de ovos por grama de fezes pela fórmula:

Nọ de ovos de S. mansoni por lâminax $1000 \mathrm{mg}$ 43,7

o que corresponde a número de ovos por lâmina vezes 23 .

Os resultados globais foram expressos pela mediana do número de ovos de $S$. mansoni 
FIG. II

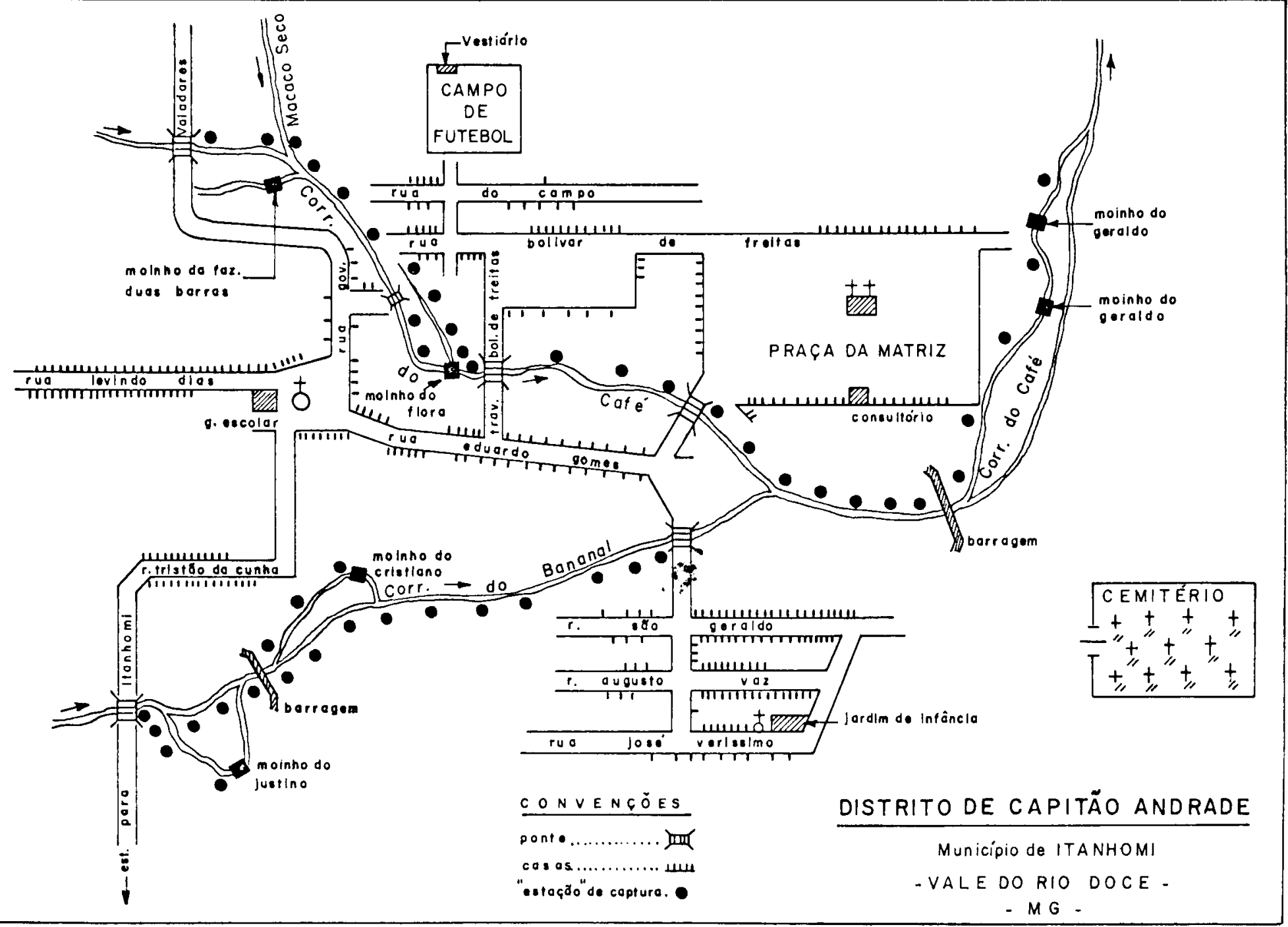




\section{GRÁFICO I}

DISTRIBUIÇÃO DOS 1480 HABITANTES DE CAPITÃO ANDRADE SEGUNDO SEXO, GRUPOS ETARIOS E ÉTNICOS.

1973
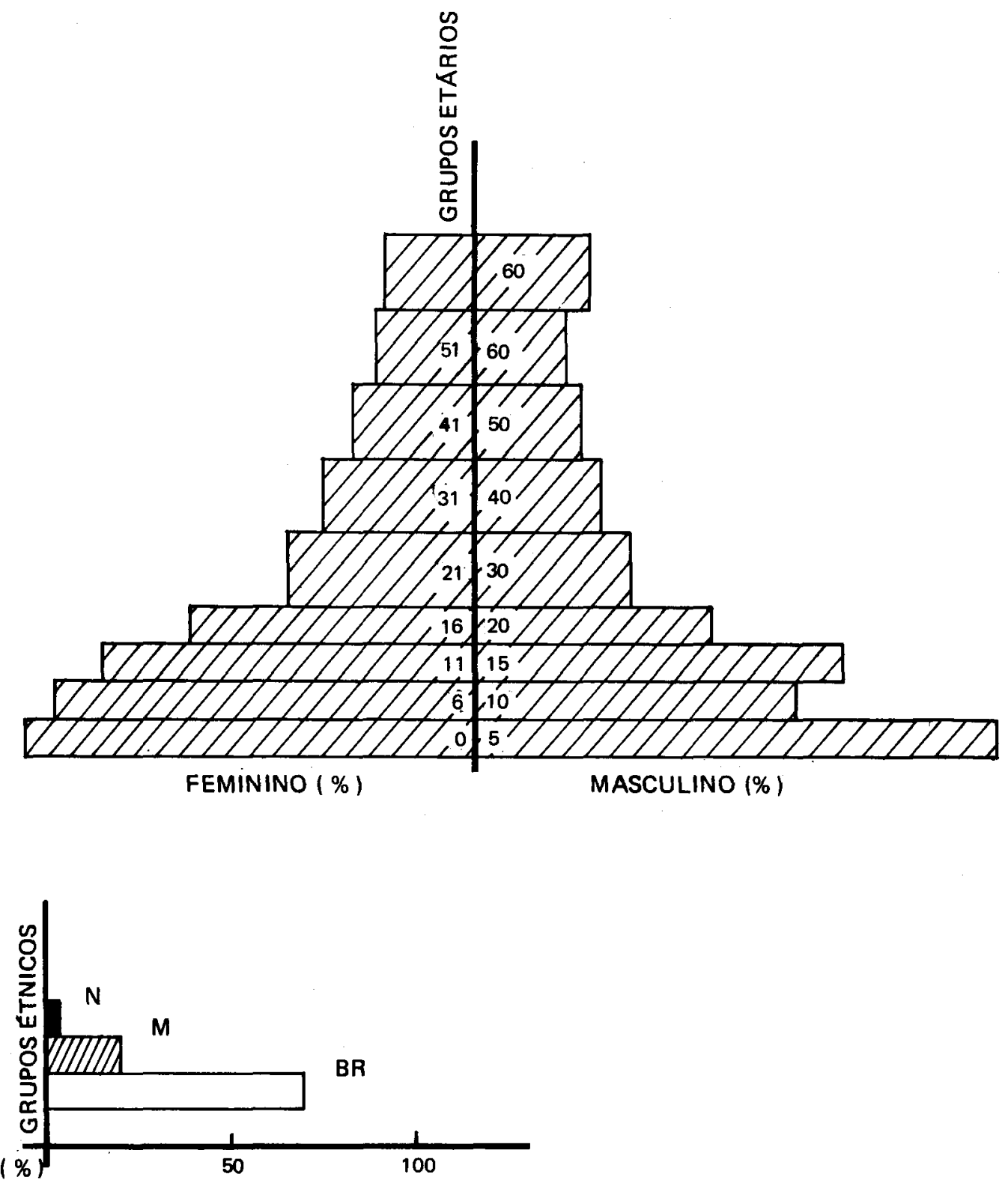

$$
\begin{aligned}
& N=\text { NEGROS } \\
& \dot{M}=\text { MESTIÇOS } \\
& \dot{B R}=\text { BRANCOS }
\end{aligned}
$$


por grama de fezes, em virtude da distribuição irregular nas contagens de ovos nas fezes.

\section{c) Intradermo-reação}

Para determinação da prevalência da infecção, além dos exames de fezes empregou-se também a intradermo-reação (IDR) com antígeno de S. mansoni em 241 pacientes dos diversos grupos etários (amostra aleatória de $16,3 \%$ da população, de acordo com a tabela de números aleatórios).

Usou-se antígeno de verme adulto, preparado pela técnica publicada por Pellegrino27. A inoculação do antígeno foi baseada na técnica padronizada por Kagan, Pellegrino e Memória12; injeção de $0,05 \mathrm{ml}$ de antígenc na região dorsal do antebraço. Depois de 15 minutos delimitava-se a área da pápula com caneta esferográfica, e, em papel de filtro umedecido com álcool, retirava-se o seu contorno para leitura posterior. De acordo com Pellegrino e Macedo 28, foram consideradas positivas as reações iguais ou superiores a um centímetro quadrado de área.

\section{d) Exame clínico}

Em 1973, por falta de local para que se improvisasse um ambulatório, realizaram-se os exames clínicos na própria residência dos pacientes, quando das visitas individuais aos $\mathbf{3 2 2}$ domicílios. Para a reavaliação clínica, em 1974, passou-se a atender os pacientes em pequeno posto médico recém construído.

Neste exame utilizou-se uma ficha clínica (Anexo I). Um dos itens indagados aos habitantes foi o tipo de contato com as águas dos córregos, considerando-se 3 tipos:

a) muito freqüente - quando o con tato era obrigatório, como ocorria aos lavradores na drenagem de água dos córregos para o cultivo de arroz; às domésticas, na limpeza de utensílios domésticos e lavagem de roupas, e às crianças em seus folguedos.

b) pouco freqüente - em caso de contatos ocasionais, como nas atividades de pesca esporádica.

c) ausente - quando havia negativa de qualquer contato com os focos.

O exame do abdomem foi realizado em decúbito dorsal e na posição de Schuster. As di- mensões do fígado e do baço foram referidas em centímetros, se palpáveis na fase inspiratória da respiração. 0 fígado, medido na linha hemiclavicular direita em relação à reborda costal direita e o baço, na linha hemiclavicular esquerda em relação à reborda costal esquerda. Especificou-se a consistência desses órgãos (mole, endurecida ou dura), além do tipo de borda (fina, romba, regular ou irregular), superfície (lisa ou nodular) e a sensibilidade (do lorosa ou indolor).

Em relação às formas clínicas, adotou-se a classificação de Pessoa e Barros 33, 34, modificada por Barbosa1, que considerou 3 tipos:

Tipo I - Esquistossomose-infeccão: com ou sem sintomas intestinais e quando presentes são pouco freqüentes, atribuíveis ou não à doença em causa.

Tipo II - Esquistossomose-doença forma hépato-intestinal: sintomatologia intestinal freqüente, particularmente com episódios disenteriformes e hepatomegalia.

Tipo III - Esquistossomose-doença forma hépato-esplênica: sintomatologia intestinal muito freqüente, principalmente com episódisenteriformes, hepatomegalia e esplenomegalia. Esta forma clínica seria classificada como descompensada, quando acompanhada de edemas, ascite e circulação colateral.

e) Estudo do hospedeiro intermediário

Os moluscos foram coletados em períodos regulares no decorrer do ano e colocados em frascos que indicavam o número da estação $\mathrm{e}$ a data da coleta. Em cada estação determinava-se a temperatura da água na ocasião da coleta.

Submeteram-se os moluscos à luz artifical, individualmente, em tampas de Borrel com água durante 60 minutos. A seguir eram exa minados íntegros sob lupa entomológica, registrando-se os caramujos que eliminavam cercárias; nos negativos aplicou-se o método de esmagamento, sendo reexaminados. Nos casos positivos transferiam-se com pipeta Pasteur algumas gotas do-material para placa de Petri, corando-se com lugol para verificação da morfologia das cercárias.

Uma amostra de caramujos foi encaminhada para classificação no "Centro para as Américas de Identificação de Caramujos Transmis- 


\section{ANEXO I - FICHA CLINICA}

NOME:

IDADE: SEXO: GRUPO ÉTNICO:

PROFISSAO: NATURALIDADE:

Tempo de permanência na área: Afastamento:

Contato: Muito freqüente:

Pouco freqüente:

Ausente:

EXAME CLINIICO:

Peso: Altura: Pressão Arterial: Ausculta cardíaca:

\begin{tabular}{|c|c|}
\hline FIGADO & 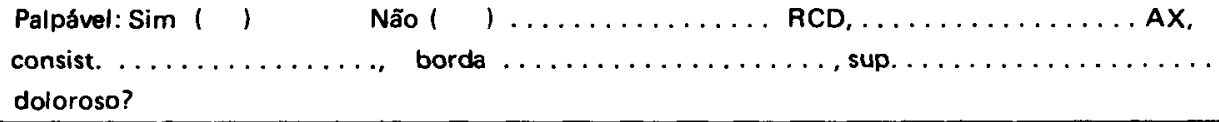 \\
\hline BAÇO & 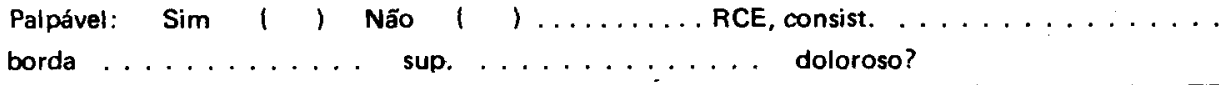 \\
\hline
\end{tabular}

RITMO INTESTINAL NOS ÚLTIMOS 6 MESES:

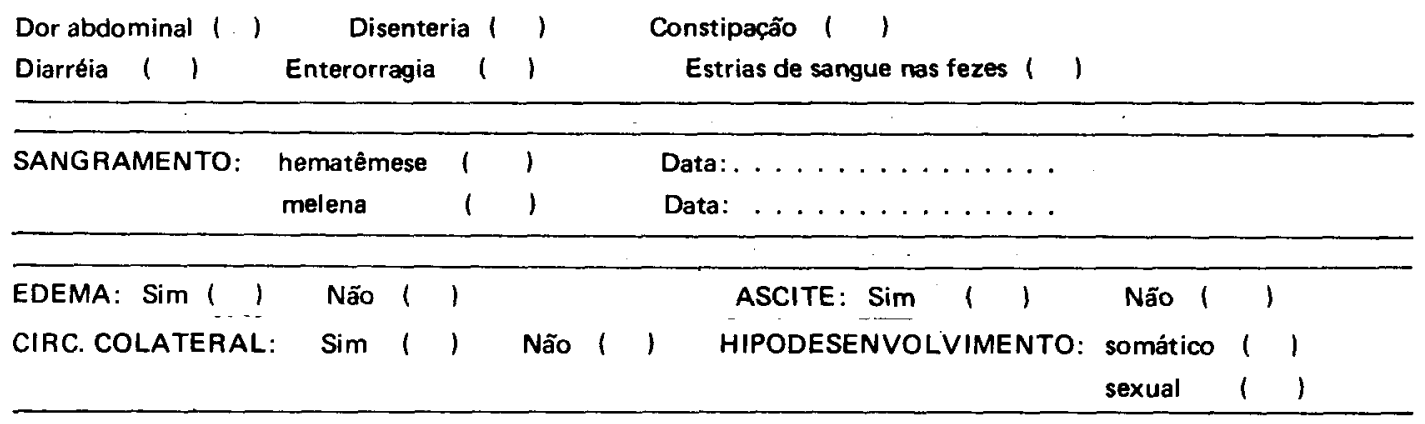

TRATAMENTo: $\sin 1$, Não 1, DROGa(s) $\ldots \ldots \ldots$ DATA: .......



\begin{tabular}{lllll}
\hline EX. FEZES: Lutz & Kato: & ovos/g & IDR: \\
\hline
\end{tabular}

OBS:

sores da Esquistossomose", dirigido pelo Professor Lobato Paraense, no Instituto Central de Biologia da Universidade de Brasília.

\section{RESULTADOS}

a) Exames coproscópicos

Na primeira etapa do trabalho (1973), foram realizados 1.234 exames de fezes $(83,3 \%$ da população), sendo 589 (47,7\%) em pacientes do sexo masculino e 645 (52,2\%) em pacientes do sexo feminino.

A prevalência da infeç̧ão pelo exame de fezes variou de $16,1 \%$ no grupo de 0 a 5 anos a $83,9 \%$ no grupo de 21 a 30 anos, decrescendo no grupo com mais de 50 anos. A prevalência para toda população estudada foi de $60,8 \%$ como pode ser verificado na Tabela $\mathrm{I}$. 
A positividade para ovos de $\mathrm{S}$. mansoni foi maior no sexo masculino $(65,8 \%)$ que no fe$\operatorname{minino}(56,3 \%)$.

Na segunda etapa (1974), dos 1234 exames realizados no ano anterior, foi possível repetir
1.050: $508 \mathrm{em}$ pacientes do sexo masculino e 542 nos de sexo feminino, obtendo-se preva lência para esquistossomose de $66,1 \%$ e $58,7 \%$, respectivamente. A positividade para os 1.050 exames de fezes executados foi de $62,3 \%$, conforme mostra a Tabela II.

TABELA I

PREVALENNCIA DA ESOUISTOSSOMOSE MANSONI ATRAVÉS DE EXAMES DE FEZES PELOS MÉTODOS DE LUTZ E OU KATO DISTRIBUIDA POR SEXO E GRUPO ETARIO CAPITÃO ANDRADE, 1973.

\begin{tabular}{|c|c|c|c|c|c|c|c|c|c|}
\hline \multirow{2}{*}{$\begin{array}{l}\text { GRUPO } \\
\text { ETARIO }\end{array}$} & \multirow[b]{2}{*}{ Nọ } & \multicolumn{2}{|c|}{ TOTAL } & \multicolumn{3}{|c|}{ MASCULINO } & \multicolumn{3}{|c|}{ FEMININO } \\
\hline & & Nọ & $\%$ & Nọ & Nọ & $\%$ & No & Nọ & $\%$ \\
\hline $0-5$ & 223 & 36 & 16,1 & 119 & 21 & 17,6 & 104 & 15 & 14,4 \\
\hline $6-10$ & 186 & 110 & 59,1 & 86 & 60 & 69,7 & 100 & 50 & 50,0 \\
\hline $11-15$ & 193 & 142 & 73,6 & 95 & 75 & 78,9 & 98 & 67 & 68,3 \\
\hline $16-20$ & 115 & 96 & 83,4 & 50 & 44 & 88,0 & 65 & 52 & 80,0 \\
\hline $21-30$ & 143 & 120 & 83,9 & 60 & 51 & 85,0 & 83 & 69 & 83,1 \\
\hline $31-40$ & 122 & 84 & 68,8 & 53 & 41 & 77,3 & 69 & 43 & 62,4 \\
\hline $41-50$ & 91 & 69 & 75,8 & 41 & 34 & 82,9 & 50 & 35 & 70,0 \\
\hline $51-60$ & 73 & 43 & 58,9 & 35 & 25 & 71,4 & 38 & 18 & 47,3 \\
\hline+60 & 88 & 50 & 56,8 & 50 & 36 & 72,0 & 38 & 14 & 36,8 \\
\hline TOTAL & 1234 & 750 & 60,8 & 589 & 387 & 65,8 & 645 & 363 & 56,3 \\
\hline
\end{tabular}

Nọ - Número de exames de fezes realizados.

TABELA ॥

PREVALENCIA DA ESQUISTOSSOMOSE MANSONI ATRAVÉS DE EXAMES CE FEZES PELOS MÉTODOS DE LUTZ E OU KATO DISTRIBUIDA POR SEXO E GRUPC ETARIO. CAPITÃO ANDRADE, 1974.

\begin{tabular}{|c|c|c|c|c|c|c|c|c|c|}
\hline \multirow{3}{*}{$\begin{array}{l}\text { GRUPO } \\
\text { ETARIO }\end{array}$} & \multicolumn{3}{|c|}{ TOTAL } & \multicolumn{3}{|c|}{ MASCULINO } & \multicolumn{3}{|c|}{ FEMININO } \\
\hline & \multirow[b]{2}{*}{ No } & \multicolumn{2}{|c|}{ POSITIVO } & \multirow[b]{2}{*}{ No } & \multicolumn{2}{|c|}{ POSITIVO } & \multirow[b]{2}{*}{ Nọ } & \multicolumn{2}{|c|}{ POSITIVO } \\
\hline & & No & $\%$ & & Nọ & $\%$ & & Nọ & $\%$ \\
\hline $0-5$ & 172 & 22 & 12,8 & 89 & 15 & 16,8 & 83 & 7 & 8,4 \\
\hline $6-10$ & 170 & 107 & 62,9 & 86 & 57 & 66,3 & 84 & 50 & 59,5 \\
\hline $11-15$ & 161 & 129 & 80,1 & 75 & 64 & 85,3 & 86 & 65 & 75,6 \\
\hline $16-20$ & 101 & 84 & 83,1 & 47 & 42 & 89,3 & 54 & 42 & 77,8 \\
\hline $21-30$ & 118 & 99 & 83,9 & 47 & 43 & 91,4 & 71 & 56 & 78,9 \\
\hline $31-40$ & 99 & 72 & 72,7 & 47 & 37 & 78,7 & 52 & 35 & 67,3 \\
\hline $41-50$ & 90 & 60 & 66,6 & 41 & 33 & 80,5 & 49 & 27 & 55,1 \\
\hline $51-60$ & 61 & 41 & 67,2 & 31 & 22 & 71,0 & 30 & 19 & 63,3 \\
\hline+60 & 78 & 40 & 51,2 & 44 & 23 & 52,3 & 34 & 17 & 50,0 \\
\hline TOTAL & 1050 & 654 & 62,3 & 508 & 336 & 66,1 & 542 & 318 & 58,7 \\
\hline
\end{tabular}

No - Número de exames de fezes realizados 
b) Intradermo-reação

Na amostra de $16,3 \%$ da população total a intradermo-reação com antígeno de S. mansoni revelou positividade de $25,6 \%$ em crianças de 0 a 5 anos de idade, elevando-se a $86,2 \%$ no grupo etário de 6 a 10 anos, até alcançar $100 \%$ nos pacientes de 16 a 20 anos. $\mathrm{Na}$ faixa de 21 a 50 anos a prevalência através da IDR oscilou entre $90,3 \%$ a $96,1 \%$, decrescendo progressivamente a partir do grupo com mais de $\mathbf{5 0}$ anos de idade. A prevalência média da positividade da IDR para esquistossomose na amostra estudada para todos os grupos etários foi de $80,8 \%$, sendo de $82,9 \%$ para o sexo masculino e $77,4 \%$ para o sexo feminino (Tabela IIII).

A análise estatística demonstrou:

1. Diferença significativa nos resultados de' prevalência da infecção esquistossomótica pela IDR entre os diversos grupos etários estudados.

$X^{2}=20,6126$ para 8 G.L. $(0,001<P<0,01)$.

2. Que não existiu diferença significativa na amostra estudada entre os resultados finais pela JDR nos sexos masculinos e feminino.

$Z=1,0659(P>0,05)$.

3. Que ocorreu diferença significativa entre a prevalência obtida por exame de fezes e a determinada pela IDR; sendo maior a prevalência da infecção através da IDR.

$Z=6,14(P>0,001)$

\section{c) Exame clínico}

Examinaram-se clinicamente 1.369 pessoas residentes na área, isto é, 92,5\% dos habitantes, não sendo possivel examinar $7,5 \%$ da população residente na comunidade em estudo.

Segundo a classificação clínica de Pessoa e Barros 33, modificada por Barbosa 1 , dos 750 pacientes com infecção esquistossomótica $61,7 \%$ foram incluídos no tipo $1,32,5 \%$ no tipo II e 5,8\% no tipo III. Neste último grupo, $5 \%$ era do sexo masculino e $0,8 \%$ do sexo feminino. (Tabela IV). Apenas um paciente de 20 anos, do sexo masculino, apresentou forma descompensada.

Dos pacientes incluídos no tipo III, 6 $(0,8 \%)$ apresentaram sinais e sintomas de hi-

TABELA III

DISTRIBUIÇÃO DA PREVALENCIA DA ESQUISTOSSOMOSE MANSONI ATRAVES DA INTRADERMO-REAÇÃO

EM AMOSTRA ALEATÓRIA DA POPULAÇÃO SEGUNDO SEXO E GRUPO ETÁRIO. CAPITÃO ANDRADE, 1973.

\begin{tabular}{|c|c|c|c|c|c|c|c|c|c|}
\hline \multirow{3}{*}{$\begin{array}{l}\text { GRUPO } \\
\text { ETARIO }\end{array}$} & \multicolumn{3}{|c|}{ TOTAL } & \multicolumn{3}{|c|}{ MASCULINO } & \multicolumn{3}{|c|}{ FEMININO } \\
\hline & \multirow[b]{2}{*}{ Nọ } & \multicolumn{2}{|c|}{ POSITIVO } & \multicolumn{3}{|c|}{ POSITIVO } & \multirow[b]{2}{*}{ Nọ } & \multicolumn{2}{|c|}{ POSITIVO } \\
\hline & & no & $\%$ & Nọ & $\overline{n !}$ & $\%$ & & no & $\%$ \\
\hline $0-5$ & 43 & 11 & 25,6 & 25 & 9 & 36,0 & 18 & 2 & 11,1 \\
\hline $6-10$ & 29 & 25 & 86,2 & 12 & 11 & 91,6 & 17 & 14 & 82,3 \\
\hline $11-15$ & 41 & 39 & 95,1 & 18 & 18 & 100,0 & 23 & 21 & 91,3 \\
\hline $16-20$ & 26 & 26 & 100,0 & 11 & 11 & 100,0 & 15 & 15 & 100,0 \\
\hline $21-30$ & 31 & 28 & 90,3 & 17 & 16 & 94,1 & 14 & 12 & 85,7 \\
\hline $31-40$ & 18 & 17 & 94,4 & 9 & 9 & 100,0 & 9 & 8 & 88,8 \\
\hline $41-50$ & 26 & 25 & 96,1 & 11 & 10 & 90,9 & 15 & 15 & 100,0 \\
\hline $51-60$ & 13 & 12 & 92,3 & 6 & 6 & 100,0 & 7 & 6 & 85,7 \\
\hline+60 & 14 & 10 & 71,4 & 8 & 7 & 87,5 & 6 & 3 & 50,0 \\
\hline TOTAL & 241 & 193 & 80,8 & 117 & 97 & 82,9 & 124 & 96 & 77,4 \\
\hline
\end{tabular}

Obs.: Nọ - Número de intradermo-reações realizadas no - número de in tradermo-reações positivas 
TABELA IV

CLASSIFICAÇÃO CLINIICA DE 750 PORTADORES DE S MANSONI DISTRIBUIDA SEGUNDO OS GRUPOS ETARIOS.

CAPITÃO ANDRADE, 1973.

\begin{tabular}{|c|c|c|c|c|c|c|c|c|}
\hline \multirow{2}{*}{$\begin{array}{l}\text { GRUPO } \\
\text { ETARIO }\end{array}$} & \multicolumn{2}{|c|}{ TOTAL } & \multicolumn{2}{|c|}{ TIPO I } & \multicolumn{2}{|c|}{ TIPO II } & \multicolumn{2}{|c|}{ TIPO III } \\
\hline & No & $\%$ & No & $\%$ & No & $\%$ & No & $\%$ \\
\hline $0-5$ & 36 & 100,0 & 18 & 50,0 & 18 & 50,0 & - & - \\
\hline $6-10$ & 110 & 100,0 & 28 & 25,4 & 78 & 70,9 & 4 & 3,6 \\
\hline $11-15$ & 142 & 100,0 & 36 & 25,3 & 85 & 59,8 & 21 & 14,8 \\
\hline $16-20$ & 96 & 100,0 & 71 & 73,9 & 19 & 19,8 & 6 & 6,2 \\
\hline $21-30$ & 120 & 100,0 & 98 & 81,6 & 15 & 12,5 & 7 & 5,8 \\
\hline $31-40$ & 66 & 100,0 & 53 & 80,3 & 11 & 16,6 & 2 & 3,0 \\
\hline $41-50$ & 69 & 100,0 & 61 & 88,4 & 6 & 8,7 & 2 & 2,8 \\
\hline $51-60$ & 52 & 100,0 & 48 & 92,3 & 4 & 7,6 & - & - \\
\hline+60 & 59 & 100,0 & 50 & 84,7 & 8 & 13,5 & 1 & 1,6 \\
\hline TOTAL & 750 & 100,0 & 463 & 61,7 & 244 & 32,5 & 43 & 5,8 \\
\hline
\end{tabular}

Obs.: № - número de paoientes portadores de $S$. mansoni.

podesenvolvimento somático e sexual: 4 do sexo masculino, de idades variáveis entre 15 e 18 anos e esplenomegalia de 4 a 9 centímetros da reborda costal esquerda; e 2 pacientes do sexo feminino, de 15 a 18 anos, ambos com esplenomegalia de 5 centímetros.

No estudo evolutivo das formas clínicas no decorrer de um ano verificou-se que 24 pacientes $(5,2 \%)$ evoluiram do tipo I ao tipo II; 14 eram do sexo masculino e 10 do sexo feminino, a maior parte deles compreendida nos grupos etários entre 5 a 15 anos de idade.

Não se observou evolução de pacientes da forma clínica tipo II para o tipo III.

Quando se tentou correlacionar a sintomatologia dos pacientes com as formas clínicas da doença, observou-se que os episódios disentéricos foram referidos em $44,9 \%$ dos individuos classificados no tipo I, em $69,2 \%$ nos de tipo II e em 92,3\% nos de tipo III. Encontrouse $24,1 \%$ de pacientes assintomáticos no tipo I, $13,8 \%$ no tipo II, não se verificando casos assintomáticos no tipo III.

Dos pacientes autóctones, 11 referiram esplenectomia nos últimos 10 anos, 9 homens e duas mulheres, o que representou um índice de esplenectomia de $1,68 \%$ na população estudada.

As indicações referidas para a cirurgia foram episódios hemorrágicos em 1,07\%, dor no hipocôndrio esquerdo em $0,45 \%$ e essa úl- tima queixa clínica associada a hipoevolutismo em $0,15 \%$.

Ocorreram 19 óbitos $(1,2 \%$ da população estudada), no decorrer de um ano: $16(2,1 \%)$ no sexo masculino e $3(0,4 \%)$ no sexo feminino; 3 abaixo de 5 anos de idade, 1 no grupo etário de 6 a 10 anos, 4 no grupo de 21 a 40 anos, 4 no de 41 a 50 anos e 7 óbitos no grupo acima de 50 anos.

Dois óbitos $(0,2 \%)$ ocorreram no pós-operatório em doentes com 21 a 38 anos que apresentavam forma hépato-esplênica. As indicações cirúrgicas surgiram depois de episódios hemorrágicos.

Nos úl timos 10 anos, 66 indivíduos, 4,54\% dos habitantes, tinham procurado tratamento especifico nas cidades vizinhas. A droga mais utilizada foi hycanthone, seguida de fuadina, stibophen, triostib, ambilhar e tártaro emético. Dos 33 pacientes submetidos a tratamento com hycanthone nos últimos dois anos, $50 \%$ apresentavam exames de fezes positivos para ovos de S. mansoni.

Em relação ao contato com as águas dos córregos, determinou-se que nas formas clínicas tipos I, II e III, respectivamente, $50,2 \%$, $89,7 \%$ e $97,7 \%$ dos pacientes tinham contato muito freqüente.

O contato pouco freqüente nos casos de tipos I, II e III foi de $49,8 \%, 10,2 \%$ e $2,3 \%$, res. pectivamente. 
d) Estudo do hospedeiro intermediário

Os planorbídeos da área foram classificados no gênero Biomphalaria espécie glabrata, com índice de infeç̧ão variável de $2,7 \%$, em setembro de 1973, a 1,5\%, em abril de 1975. (Gráfico II).

\section{DISCUSSĀO}

a) Prevalência da infecção esquistossomótica

Em nossa experiência, a infecção esquistossomótica atinge baixos percentuais nos primeiros anos de vida, elevando-se gradativamente

GRAFICO ॥

INDICES DE INFECÇÃO DE B GLABRATA SEGUNDO AS VARIAÇŌES NA ESTAÇÃO DE NUMERO UM NO CÓRREGO DO CAFÉ.

CAPITÃO ANDRADE, $1973-1975$.

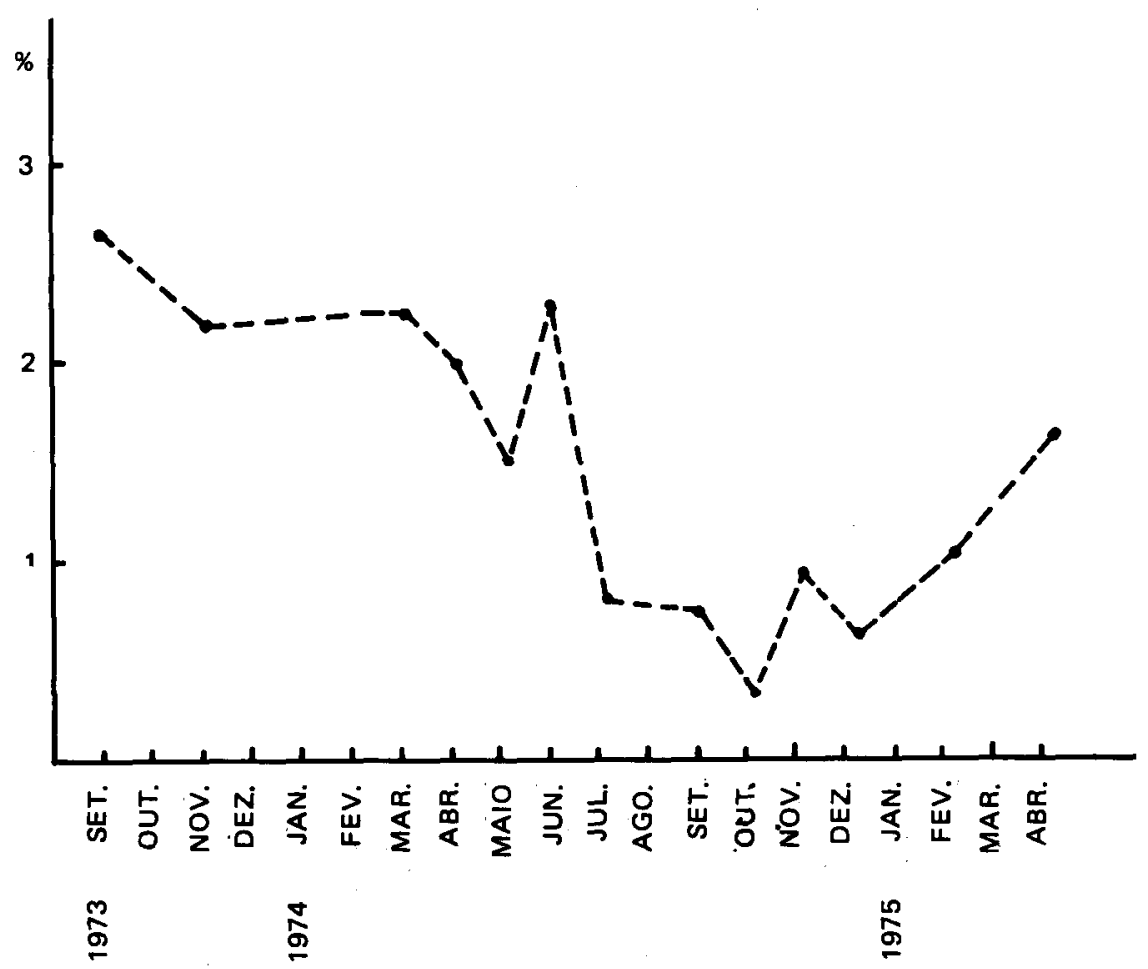


até alcançar níveis máximos nos grupos etários entre 16 e 30 anos. Esses resultados confirmam os dados de vários autores que obtiveram maior prevalência da esquistossomose em jovens nas zonas endêmicas brasileiras (Martins e Versiani23), (Pessoa ${ }^{32}$ ), (Barbosa).

Observamos predomínio de prevalência da infecção, pelo exame de fezes, no sexo masculino, o que tem sido divulgado nas publicações de Dias ${ }^{8}$. Numa das localidades de Pernambuco, Barbosa ${ }^{1}$ relata predominância da infecção em mulheres. Entretanto, Brener e Mourão 3 não se referem à diferença de prevalência entre os sexos.

Nos adultos a maior positividade da infecção que encontramos nos homens dever-seia ao prepara das lavouras, quando são obrigados a drenarem água dos córregos para o plantio de arroz e nas pescarias, enquanto buscam momentos de distraçăo. Essa variação de prevalência por sexo em cada região parece depender diretamente das atividades exercidas pelos indivíduos.

No estudo de prevalência da esquistossomose a positividade média de $80,2 \%$ revelada pela intradermo-reação demonstrou maior sensibilidade de detectar a infecção do que o exame de fezes, confirmando o que tem sido descrito (Kagan, Pellegrino e Memória12.

A positividade da reação intradérmica variou de modo significativo com os grupos etários, apresentando-se mais elevada em adultos do que nas crianças, de acordo com as referências de Pessoa e Barros 34.

Năo houve variação significativa da reação entre os sexos masculino e feminino na amostra estudada, de modo semelhante ao que observou Barbosa1

Verificou-se que a reação intradérmica foi menos sensível em detectar infecção em criancas abaixo de 5 anos de idade do que em grupos etários maiores, embora as reações fossem realizadas na região dorsal do antebraço (em que os resultados são superiores aos da região ventral), como preconizam Pellegrino e Memória29.

A razão da diferença de sensibilidade da reação entre crianças e adultos parece dever-se à necessidade de muitos anos de infecção, para que se desenvolva uma reação positiva, como afirmam Kloetzel e Silva17. Entretanto, apesar da menor sensibilidade da reação em crianças abaixo de 5 anos, em nossas observações nesse grupo ela mostrou-se superior $(25,5 \%)$ ao exame de fezes $(16,1 \%)$, na determinação da prevalência da infecção.

Um fato que merece apreciação é o de 9 crianças $(2,5 \%)$ com idades entre 4 e 9 anos que apresentavam exames de fezes positivos e reações intradérmicas negativas em 1973 a 1974. Barbosa1 citou essa mesma ocorrência em $6,5 \%$ de indivíduos de 4 localidades de Pernambuco, sobretudo na faixa etária entre 1 e 9 anos. Cogitamos sobre a possibilidade daquelas crianças estarem incluídas no grupo de $6 \%$ de indivíduos que não reagem ao antígeno de verme adulto, empregado na presente pesquisa, mas apenas ao antígeno cercariano (Kagan, Pellegrino e Memória12).

\section{b) Formas clínicas}

A maior parte dos individuos infectados $(61,7 \%)$ foi classificada no tipo 1 , de modo semelhante aos dados de Brener e Mourão3. Os $\mathbf{5 , 8 \%}$ dos indivíduos do tipo II predominaram no grupo etário de 11 a 15 anos, informação esta coincidente com as de Rodrigues da Silva 38 . No entanto, Barbosa ${ }^{1}$ refere-se a essa forma clínica em faixas etárias acima de 15 anos e em pacientes com idade superior a 50 anos. Brener -e Mourão 4 descreveram em inquéritos distribuição uniforme dos índices de esplenomegalia nos diversos grupos ¿tá rios, "com exceção dos grupos abaixo de 4 anos e acima de 40 anos de idade.

Verificamos maior índice de hépato-esplenomegalia nos homens do que nas mulheres, o que tem sido referido por Rodrigues da Silva38, Brener e Mourão4. Em localidades de Sergipe (Pessoa e Barros 33 , e de Pernambuco (Kloetzel17), não obtiveram essa distinção de esplenomegalia entre os sexos.

Verificamos em Capitão Andrade que os pacientes com formas clínicas graves, tipos II e III, apresentaram contato mais freqüente com os córregos do que os classificados no tipo I; assim, podemos estabelecer na comunidade uma correlação entre gravidade clínica e maior exposição aos focos, fato que é ressaltado por $S \operatorname{cott} 40$

\section{c) Estudo do hospedeiro intermediário}

Embora só se encontrassem B. glabrata infectados provenientes da estação de númern 
um, no córrego do Café, conhece-se a possibilidade de as cercárias promoverem infecção, até uma distância de um quilômetro do foco de moluscos infectantes (Paraense e Santos 26. Segundo esses autores, o fenômeno decorreria/da ação de correntes de retorno formadas pelas ondas de translação, dispersando cercárias pelas águas do córrego.

Os caramujos, coletados na estação de número um em períodos mensais ou bimensais, estavam infectados; assim, a área pode ser caracterizada como um foco de tranismissão ativa.

\section{CONCLUSÕES}

O estudo sobre a morbidade da esquistossomose mansoni realizado no distrito de Capitão Andrade, munićpio de Itanhomi, Vale do Rio Doce, Minas Gerais, no período de 1973 a 1974 possibilitou as seguintes çonclusões:

1. A área pode ser considerada hiperendêmica de esquistossomose mansoni em decorrência da prevalência média acima de $60 \%$ da população total nos dois anos consecutivos de estudo. Além de apresentar elevada morbidade, estimada pelos $5,8 \%$ de pacientes com formas hépato-esplênicas e pela passagem progressiva de $5,2 \%$ dos casos do tipo I para o tipo II no período de 1 ano.

2. A prevalência da infecção esquistossomótica através de exames de fezes foi de $16,1 \%$ no grupo etário de 0 a 5 anos, elevando-se gradativamente até atingir $83,9 \%$ no grupo de 21 a 30 anos, decrescendo inversamente à idade a partir de então. Observou-se predomínio de prevalência do sexo masculino sobre o feminino em ambas as etapas do estudo.

3. A prevalência da infecção através da intradermo-reação com antígeno de verme adulto variou de $25,6 \%$ em crianças de 0 a 5 anos de idade a $100 \%$ em jovens de 16 a 20 anos, decrescendo a partir de $\mathbf{5 0}$ anos de idade. A positividade da infecção pela intradermo-reação foi superior à determinada pelo exame de fezes e não se demonstrou diferença significativa de positividade pela reação entre os sexos.

4. A intensa associação de outros parasitos intestinais com o $S$. mansoni na comunidade estudada não permite que se relacione a sintomatologia intestinal referida pelos pacientes ao parasitismo exclusivo pelo verme, com ex- ceção do sintoma enterorragia, por ter ocorrido com maior freqüência nos habitantes portadores do $S$. mansoni, não sendo praticamente relatado em sua ausência.

5. Verificou-se hipoevolutismo somático e sexual em $0,8 \%$ dos pacientes hépato-esplênicos com idades variáveis entre 15 e 18 anos e uma notável predisposição familiar à esplenomegalia esquistossomótica, sugerindo pesquisas mais aprofundadas quanto a este aspecto.

6. A Biomphalaria glabrata é o vetor da esquistossomose mansoni na área em estudo e apesar de os moluscos infectados serem provenientes de somente uma das estações pesquisadas, é possível a existência de cercárias e a transmissão da doença em outros locais dos córregos peló fenômeno de "dispersão cercariana" a partir de um determinado foco.

\section{SUMMARY}

A two-years cross-sectional study of schistosomiasis mansoni in a rural community of the county of Itanhomi (State of Minas Gerais, Brasil) was performed. Particular attention was given to morbidity rates in 1,480 inhabitants, sanitary facilities, and intermediate host infection rates, in view of a later longitudinal work at the same region.

Figures of prevalence over 60 percent in 1973 and 1974, and a 5.8 percent of patients with severe forms of the disease lliver and spleen enlargement), suggest the high endemicity of schistosomiasis in the area.

Distribution of the infected population as to the clinical forms of the disease were as follows: 61.7 percent with subclinical infections; 32.5 percent with hepatointestinal form; and 5.8 percent with liver and spleen, enlargement (including 0.8 percent of teenagers with delayed sexual maturation and physical growth).

Strong corre/ation was observed between severe cases in 11 to 15 years old males and greater exposition to the intermediate host (Biomphalaria glabrata) foci, and higher infection rates as shown by the average number of eggs of S. mansoni per gram of faeces.

\section{REFERÊNCIAS BIBLIOGRÁFICAS}

1. BARBOSA, F.A.S.: Mordidade da Esquistossomose.: Rev. Bras. Malar., Número Especial, 3-159, 1966. 
2. BINA, J.C. \&PRATA, A.: Hycanthone no tratamento de esquistossomose em uma área rural com baixo índice de transmissão da doença. Gaz. Méd. Bahia, 70(2) : 127-130, 1970.

3. BRENER, Z. \& MOURÃO, O.G.: Inquéritos clínico-epidemiológicos em focos endêmicos de Esquistossomose mansoni em Minas Gerais. Rev. Bras. Malar., 8(4) : 519-526, 1956a.

4. BRENER, Z \& MOURÃO, O.G.: Observações sobre a forma hépato-esplênica da Esquistossomose mansoni em Minas Gerais. Rev. Bras. Malar., 8(3) : 511-517, 1956b.

5. CNPq: Estudos Iongitudinais da Esquistossomose: Objetivos, conceituação e metodologia. Documento do Grupo de Estudos sobre Esquistossomose mansoni: Curitiba, fevereiro de 1974. 35p.

6. CONCEIÇÃO, M.J.; NOLASCO, P.;STEINER, J. \& COURA, J.R.: Estudo clinico sobre Esquistossomose mansoni em área rural do município de Itanhomi, Minas Gerais. Anais do IX Congresso da Sociedade Brasileira de Medicina Tropical. Fortaleza, 1973. Resumo no 123.

7. COURA, J.R.; WANKE, B.; FIGUEIREDO, N. \& ARGENTO, C.A.: Evolutive pattern of schistosomiasis and lifespan of $S$. mansoni in patients living in non-endemic area in Brazil. Rev. Soc. Bras. Med. Trop., 8(4) : 193-198, 1974.

8. DIAS, E.: Estudos preliminares sobre a Esquistossomose mansoni no município de Bambuí, Estado de Minas Gerais. Rev. Bras. Malar., 5(3): 211-215, 1953.

9. FREITAS, C.A.: Situação atual da Esquistossomose no Brasil. Rev. Bras. Malar., 24(1/4) : 3-63, 1972.

10. FUNDAÇÃO IBGE: Sinopse preliminar do
Censo Demográfico. 89 Recenseamento geral - 1970. Rio de Janeiro, 1970.

11. HOFFMAN, W.A.; PONS, J.A. \& JANER, J.L.: The sedimentation concentration method in Schistosomiasis mansoni. Puerto Rico J. Publ. H/th., 9(3): 283-291, 1934.

12. KAGAN, I.G.; PELLEGRINO, J. \&MEMORIA, J.M.P.: Studies on the standartization of the Intradermal test for the diagnosis of Bilharziasis. Amer J. Trop. Med. Hyg., 10(2) : 200-207, 1961.

13. KATO, K.: A correct application of the thick-smear technique with cellophane paper cover. A pamphlet. 9p., 1960 (In japanese). IN KOMIYA, Y. \& KOBAYASHI, A. - Evaluation of Kato thick-smear technique with a cellophane cover for helminth eggs in feces. Japan. J. Med. Sci. Biol., 19(1): 59-64, 1966.

14. KATZ, N.; ANTUNES, C.M.F.; ANDRADE, R.M.; PELLEGRINO, J. \& COELHO, P.M.Z.: An attempt to control Schistosomiasis mansoni in an endemic area by combyning clinical treatment and molluscicide application. J. Parasit., 56(4) : 434, 1970. Section II, Part 2 of 3 Parts.

15. KATZ, N. \&BRENER, Z.: Evolução clínica de 112 casos de Esquistossomose mansoni observados após 10 anos de permanência em focos endêmicos de Minas Gerais. Rev. Inst. Med. Trop. S. Paulo, 8(3) : 139-142, 1966.

16. KATZ, N.: CHAVES, A. \& PELLEGRINO, J.: A simple device for quantitative stool thick-smear technique in Schistosomiasjs mansoni. Rev. Inst. Med. Trop. S. Paulo, 14(6) : 397-400, 1972.

17. KLOETZEL, K. \& RODRIGUES DA SILVA, J.: Schistosomiasis mansoni acquired in adulthood: behavior of eggs. 
counts and the Intradermal test. Amer. J. Trop. Med. Hyg., 16(2) : 167-169, 1967.

18. LEWIS, E.M.;SANTOS, M.L.;ANTUNES, N.; CONCEIÇÃO, M.J. \& COURA, J. R.: Prevalência e morbidade da Esquistossomose mansoni em área urbana do município de Itanhomi, Minas Gerais. Comunicação Pessoal, 1974.

19. LUTZ, A.: Observações sobre a evolução do "Schistosomum mansoni". Brasilméd., 30(49) : 385-387, 1916.

20. LUTZ, A.: O Schistosomum mansoni e a Schistosomatose segundo observações feitas no Brasil. Mem. Inst. Osw. Cruz, 11(1) : 121-155, 1919.

21. LUTZ, A. \& PENNA, O.: Estudos sobre a Schistosomatose feitos no norte do Brasil por uma comissão do Instituto Oswaldo Cruz. Mem. Inst. Osw. Cruz, $10(1): 83-94,1918$.

22. MACIEL, H.: O problema da Schistosomose intestinal no Brasil. Arch. Hoso. Mar., 2(3) : 183-187, 1925.

23. MARTINS, A.V. \&VERSIANI, W.: Schistosomose mansoni no norte de Minas Gerais. Brasil-méd., 52(36) :812-816, 1938.

24. MEIRA, J.A.: Esquistosomíase mansonisubsídio ao estudo de sua incidência e distribuição geográfica no Brasil. Arch. Fac. Hig. S. Paulo, 1(1) :5-146, 1947.

25. MENEZES, A.P. \& COURA, J.R. Prevalência e morbidade da Esquistossomose mansônica em área urbana do município de Riachuelo, Sergipe. Anais do X.I Congresso da Sociedade Brasileira de Medicina Tropical. Rio de Janeiro, fevereiro de 1975.

26. PARAENSE, W.L. \& SANTOS, J.M.: Um ano de observações sobre Esquistossomose em Planorbídeos da Lagoa Santa. Rev. Bras. Malar., 5(3): 255269, 1953.
27. PELLEGRINO, J.: Diagnóstico de laboratório da Esquistossomose mansoni. Métodos Imunológicos. Rev. Bras. Malar., 2(2/3) : 507-551, 1959.

28. PELLEGRINO, J. \& MACEDO, D.G.: Novo critério de leitura da reação intradérmica na Esquistossomose. Rev. Bras. Malar., 8(3) : 499-509. 1956.

29. PELLEGRINO, J. \& MEMORIA, J.M.P.: $A$ reação intradérmica na Esquistossomose mansoni. III. Influência da idade, sexo, cor e local da reação. Rev. Inst. Med. Trop. S. Paulo, 2(4) : 218-223, 1960 .

30. PELLON, A.B. \& TEIXEIRA, I.: I. Distribuição geográfica da Esquistossomose mansônica no Brasil. Publicação da Divisão de Organização Sanitária do Ministério da Saúde. Rio de Janeiro, 1953.

31. PELLON, A.B. \& TEIXEIRA, I.: O inquérito helmintológico escolar em cinco Estados das regiões leste, sul e centro-oeste. Publicação da Divisão de Organização Sanitária do Ministério da Saúde. Rio de Janeiro. 1953.

32. PESSOA, S.B.: Epidemiologia. Rev. Bras. Malar., 8(4) : 565-594, 1956.

33. PESSOA, S.B. ¿ BARROS, P.R.: Notas sobre a Epidemiologia da Esquistossomose mansônica no Estado de Sergipe. Rev. Med. Cir. S. Paulo, 13(4) : 147-154, 1953a.

34. PESSOA, S.B. \& BAROS, P.R.: Sobre o diagnóstico da Esquistossomose mansônica na infância pela Intradermo-reação com antígeno de esquistossomos adultos. Hospital. 43(1): 19-25, 1953b.

35. PIRAJÁ DA SILVA, M.: Contribuição para o estudo da Schistosomíase na Bahia. Brasil-Méd., 22(29) : 281-283, 1908.

36. PIRAJA DA SILVA, M.: Contribution to 
the study of Schistosomiasis in Bahia. J. Trop. Med. Hyg., 12(11) : 159-163, 1909.

37. PRATA, A. \& BINA, J.C.: Development of the hepatosplenic form of Schistosomiais. Gaz. Méd. Bahia, 68(2) : 49$60,1968$.

38. RODRIGUES DA SILVA, J.: Estudo clínico da Esquistossomose mansoni; Doença de Manson - Pirajá da Silva. TESE. Faculdade Nacional de Medi- cina da U.B., Rio de Janeiro, 1949, 452p.

39. SANTOS, M.L. \& COURA, J.R.: Prevalência e morbidade da Esquistossomose mansoni em área urbana do Município de Padre Paraíso, Minas Gerais. Reiatório apresentado ao CNPq, 1976.

40. SCOTT, J.A.: Schistosomiasis in Venezuela. Amer. J. Hyg., 35(3) : 337-366, 1942. 